\section{Pros and Cons of Low Voltage SEM EDX Elemental Analysis}

Ed Boyes

DuPont Company

edward.d.boyes@usa.dupont.com

It was Castaing (1) who many years ago lamented "First I realized that in massive samples which concerned the metallurgists I would have to give up the splendid resolving power that I had cheerfully envisaged, as I became aware of the terrific path that my electrons would perform haphazardly in the sample before agreeing to stop. I had to limit my ambitions to analyzing volumes of a few cubic micrometers. This was a big disappointment...". In the early days most instruments for imaging and microanalysis of bulk samples were restricted to operating only at relatively high beam energies ( $-30 \mathrm{kV}$ ) and for many (and perhaps too many) years this becarne the accepted operating procedure. However, we now know there are substantial advantages (pros) in using lower, and in some cases much lower, voltages; but also some significant limitations (cons). So what is possible?

The key is that at lower beam energy the electron penetration range into the sample is reduced greatly. For beam energy $E_{0}$ the range ( $R$ ) can be approximated by the Bethe equation (2) with $R \propto$ $E_{0}^{5 / 3}$. Changing the beam energy from $20 \mathrm{kV}$ to $5 \mathrm{kV}$ typically reduces the range by $10 x$ and the excited volume $\left(\alpha E^{5}\right.$ ) by $1000 x$ for the same $x$-ray line energy. The advantages are even greater at higher voltage ratios, e.g $\sim 100 \mathrm{x}$ and $\sim 1,000,000 \mathrm{x}$ respectively at $1.5 \mathrm{kV}$. Critically this moves electron microbeam analysis of bulk samples from resolutions at and above 1 micrometer (in this respect the laws of physics remain the same over the past half century), down into

\section{EDS of 0.1um Thin Film of Al on Si Substrate}

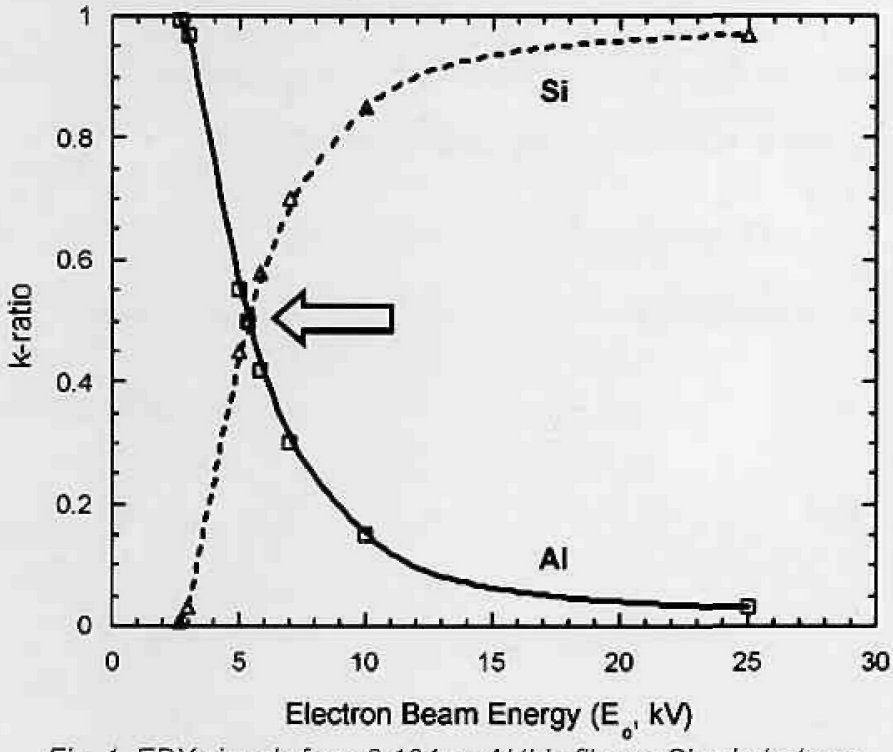

Fig. 1. EDX signals from $0.104 \mathrm{um}$ Al thin film on Si substrate as a function of beam energy $(\mathrm{kV})$.

the exciting sub-micron $(1-0.1 \mu \mathrm{m})$ and nanometer ranges. Many application technologies, including semiconductor microfabrications and pigment and fiber properties, have critical dimensions $\sim 0.1-0.25 \mu \mathrm{m}$ determined by the half wavelength of blue light or soft UV radiation. Low voltage analysis is a key component of defect review tools for these production processes; and increasingly also a powerful research tool in the chemical, materials (3) and biological (4) sciences where many problems are on a similar length scale.
The previous wide gap between high voltage analysis u rupusi alpd representative wide area bulk samples in the electron microprobie or SEM at $>1 \mu \mathrm{m}$ and thin section STEM for feature sizes $<0.1 \mu \mathrm{m}$ is finally closed. In favorable circumstances, such as the analysis $\delta f$ carbon overlayers at $1.5 \mathrm{kV}$, or Ta at $5 \mathrm{kV}$, the need for thin sectio $\bar{S}$ is pushed back with demonstrated LVEDX SEM detection sensitivities down to $<2 \mathrm{~nm}$ for thin surface films (5). In addition, it is much easier and quicker to look at a surface film directly in plan viekw than to have to make a cross-section sample (which is also genêrally much easier for SEM than (S)TEM). Fortunately, the method is not too sensitive. With careful specimen handling thin surfage films from oxidation in air (e.g. on freshly plasma cleaned $\mathrm{Cu}$ ) to Incident Electron Beam Energy (kV)

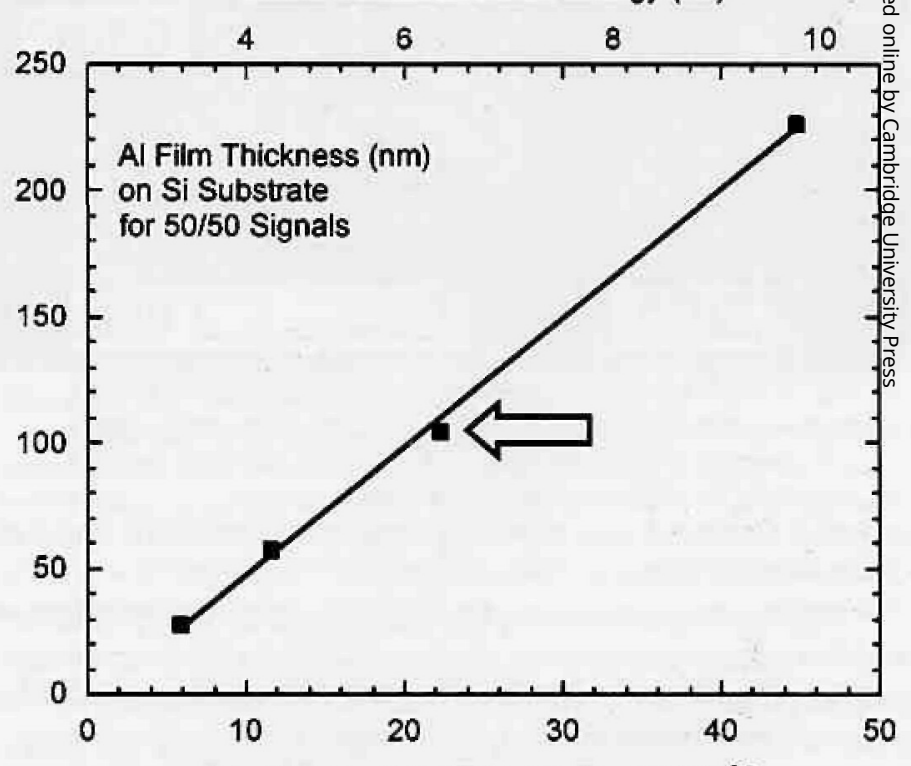

Bethe Power Law Electron Energy $\left(\mathrm{kV}^{5 / 3}\right)$

Fig. 2. Equal (Al, Si) EDX signals from Al thin films of various thickness on Si substrates with $\mathrm{kV}$ - the arrowed value is the $50 / 50$ data transferred from Fig. 1

not create undue problems. For samples heterogeneous on a fine sub-micron scale the effect of geometry on the reported chemistry can be much greater than the traditional ZAF $x$-ray corrections. Fig. 1 shows the measured k-ratios for a $0.104 \mu \mathrm{m}$ thin film of $\mathrm{Al}$ on a Si wafer as a function of electron beam energy. Many of the properties of these two materials are similar and we can think of this as being a physically homogeneous sample with a buried chemical interface parallel to the surface. Sets of such data for different film thicknesses can be interpreted to show good agreement with the Bethe equation (Fig.2). The arrows indicate the point of coincidence between Figs 1 and 2 . The improvements in volumetric sensitivity extend well down into the nanometer dimension range and there is a corresponding dramatic improvement in the spatial resolution of $\mathrm{x}$-ray maps at low voltages. Fig $3\left(\mathrm{ZrO}_{2} / \mathrm{SiO}_{2}\right.$ composite at $\left.5 \mathrm{kV}\right)$ includes features down to $50 \mathrm{~nm}$ or less in size recorded at an original magnification of $15,000 \mathrm{x}$ and far removed from the prior art. The special DuPont/Hitachi S5000SPX FESEM used here also provides $\sim 1 \mathrm{~nm}(2.5 \mathrm{x}$ ) electron image resolution at $1 \mathrm{kV}$ and superior $(0.3 \mathrm{sr}$ or 15x) EDX sensitivity. A potential downside of low voltage analysis is the increased energy density in the sample. This is the origin of the improved analytical resolution (pro) but it can also have undesirable (con) side effects including damage creation in sensitive samples. The total injected energy per $x$-ray count generated is increased only modestly $(-2 x)$ with $\mathrm{kV}$ and on a temporal or rate basis it is 


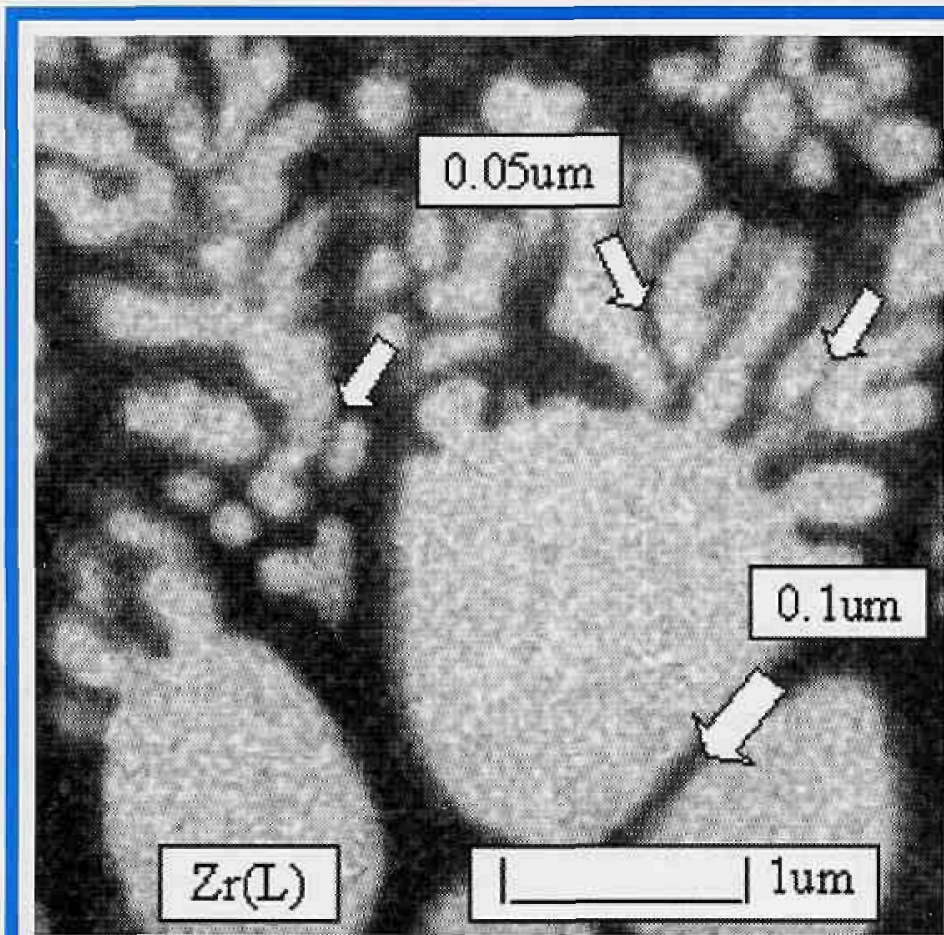

Fig. 3. High resolution chemical image map of $\mathrm{ZrO}_{2}$ in $\mathrm{SiO}_{2}$ matrix using $\mathrm{Zr}(L) \mathrm{x}$-ray line at $5 \mathrm{kV}, 0.2 n \mathrm{~A} / 2 n \mathrm{n}$ probe, $256 \mathrm{x}$ 256 spectral image recorded in 11 minutes live time on SPX system at $15,000 \times$ original magnification.

reduced. Contamination is also probably more prevalent, and it is certainly more visible, at low voltages.

The corollary of the limited interaction range for $x$-ray production with low voltage beams is the similar reduction in the $x$-ray escape path length, also by factors of $>10 x$. This is generally much more important than differences in take-off angle. It can lead to a significant reduction, and in many cases the effective elimination, of matrix absorption (A) and fluorescence $(F)$ in x-ray corrections. In this regard, but not in others $(Z)$, there are some basic similarities to thin section corrections in the (S)TEM (6). However, in the SEM the "thin section" is created in the context of the surface of the wide area native sample by restricted penetration of the electron beam into the bulk, without the physical separation or reduction required for (S)TEM. Light element analysis is generally much improved, with better and more proportionate low $Z$ representation, in spectra obtained at low beam voltages.

OK, if low voltages have all these wonderful advantages (pros) why was it not used much more widely and earlier? Well, there are some serious problems/snags (cons) too. Firstly, let us consider the fundamental physics. To excite an $x$-ray line with energy $E_{x}$ requires we use a beam energy $E_{0}$ at least $1.3 x E x$; or an over voltage $U=E_{0}$ $E_{x}>1.3$. There needs to be available a characteristic $x$-ray line at an accessible (for a signal) and useful (low enough for the application penetration range) energy. It should also have a workable fluorescent yield; for example with a $5 \mathrm{kV}$ beam $\mathrm{Cu}(\mathrm{L})$ does but $\mathrm{Ti}(\mathrm{L})$ is problematic. Secondly, the peak to background $(P / B)$ ratios and kilocounts per second per nanoamp (kcps/nA) both decline significantly as the beam energy is reduced. For $S i(K)$ the factor is $\sim 6 \mathrm{x}$ between $20 \mathrm{kV}$ and $5 \mathrm{kV}$ (fig. 4 ). This reduces the actual mass sensitivity improvement due to geometry from $\sim 1000 x$ to $-150 x$. Not catastrophic, in fact very useful; but not quite as good as is first promised. In a new instrument (DuPont/Hitachi S5000SPX FESEM) we have more than compensated for the loss in kcps/nA by improving the EDX detection sensitivity by $\sim 15 \mathrm{x}$ over previous practice. This would apply across the $k V$ range, except the $50 \mathrm{kcps} / \mathrm{nA}$ and $2 \mathrm{~nm}$ probe with $\mathrm{Al}$ at $5 \mathrm{kV}$, and $10 \mathrm{kcps}$ with thie $0.2 n A$ we prefer, translates into an unmanageable $>1 \mathrm{Mcps} / \mathrm{nA}$ for $\mathrm{Pt}$ at $30 \mathrm{KV}$. By the way, it is clear signal strength (counts) matters more than a few eV energy resolution in low voltage analysis anid larger area EDX detectors ( $30 \mathrm{~mm}^{2}$ etc.) are always preferred with FESEMs. Light elements in a heavier matrix will always be better represented in low voltage spectra irrespective of lateral resolutie because of the reduction in matrix absorption and fluorescence. Thirdly, at lower analysis voltages, the corrections for $Z$ becor a strong function of the exact accelerating voltage translated fo $_{0}$ the sample (so no charging, please). For each system (elemenहैmicroscope + detector etc.) this should be a one time determinatiōn using simple elemental standards or equivalent calculations. This"is much more manageable than the alternative need at high voltag for large, variable (including sensitivity to local take-off angle) añ.d. complex $\mathrm{A}$ and $\mathrm{F}$ corrections ( $17 \times$ for $\mathrm{O}$ in $\mathrm{SiO}_{2}$ at $30 \mathrm{kV}$ ) at ever $\mathrm{y}$ analysis point. Depending on the detector characteristics, $\mathrm{SiO}_{2}$ analyzes correctly below $10 \mathrm{kV}$. The latter value is for a perfect detector - which does not exist (not close, yet) - and $3-5 \mathrm{kV}$ is typical for a good lo-Z system today.

It helps, especially for unknown samples, if the majority of elements can be analyzed at one beam voltage and $5 \mathrm{kV}$ fulfills this need with only a few exceptions; the most important of which is Barium. In principle $7 \mathrm{kV}$ covers everything; but as always with differing yields. At voltages in the $5-7 \mathrm{kV}$ range conductive coatings are often required to stabilize conditions at the specimen surface. We currently use thin $(2-5 \mathrm{~nm})$ sputtered or evaporated Carbon and this is on the verge of detection at $5 \mathrm{kV}$. At useful thickness $(>5 \mathrm{~nm})$ metal coatings produce too many overlapping $x$-ray lines. This is a general problem even without a contribution from the coating be-

Si Wafer EDS Sensitivity and P/B as F(Eo, U)

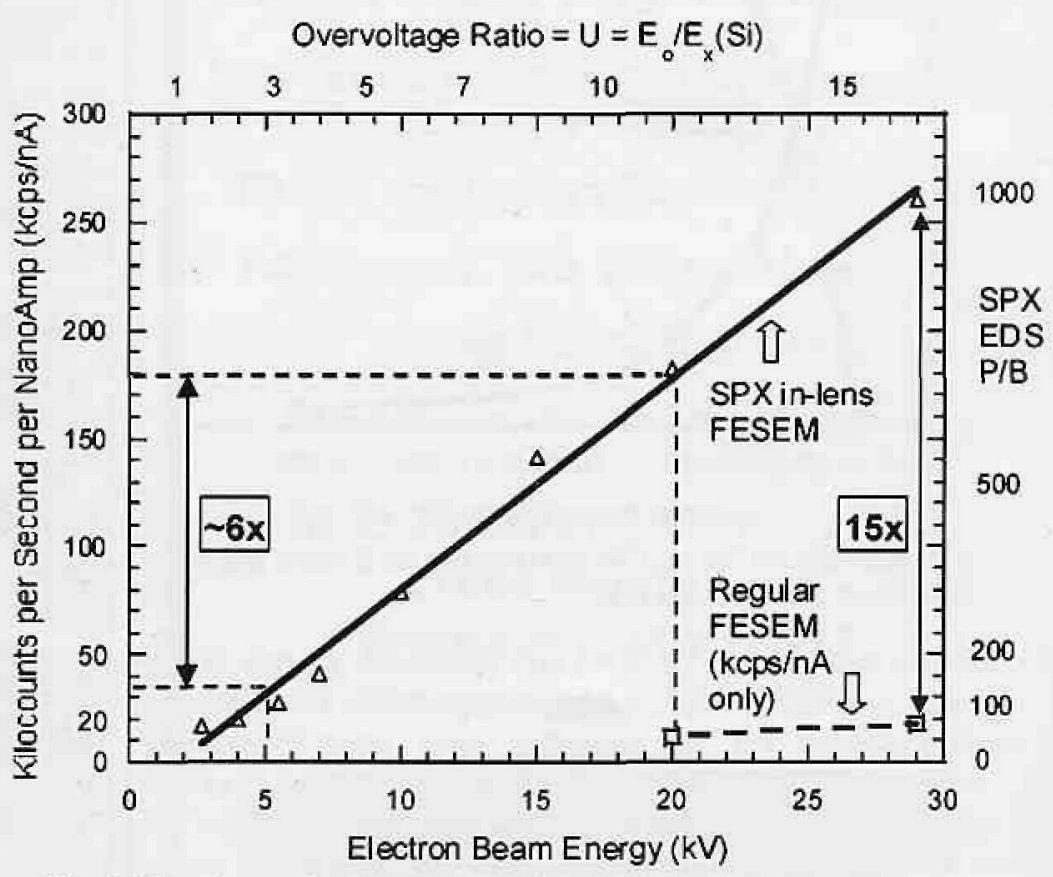

Fig. 4. Experimental $x$-ray yield and $P / B$ ratio for Si with $k V$. Values from special SPX system but the slope with $k V$ is universal. 


\section{OOInInG Events}

Microscopy NZ Conference

February 10-14, 2003, Wellington, NZ

peter.smith@agresearch.co.nz

TMS

March 2-6, 2003, San Diego, CA

ckobert@tms.org

$\checkmark \quad$ PITTCON 2003

March 9-14, 2003, Orlando, FL

www.pittcon.org

$\checkmark \quad$ Mirage 2003

March 10-12, 2003, Rocquencourt, France www.telin.rug.ac.be/mirage2003

$\checkmark \quad$ Histochemical Society Annual Meeting 2003

April 11-15, 2003, San Diego, CA

johc@u.washington.edu

$\checkmark \quad$ Experimental Biology

April 12-16, 2003, San Diego, CA

seb@sebiology.org

$\checkmark \quad$ Scanning 2003

May 3-5, 2003, San Diego, CA

www.scanning.org

$\checkmark \quad$ American Society for Microbiology

May 18-22, 2003, Washington, DC

www.asmusa.org

$\checkmark$ Microscopical Society of Canada

June 4-6, 2003, Vancouver, BC, Canada

ech@unixg.ubc.ca

$\checkmark \quad$ Lehigh Microscopy Schools

June 8, Introduction to SEM \& EDS

June 9-13, SEM \& X-ray Microanalysis

June 16-20, Advanced SEM

June 16-20, Quantitative X-ray Microanalysis

June 16-20, Analytical TEM

June 16-19, Characterization of Nanostructures

June 17-19, TEM Specimen Preparation

June 17-19 Atomic Force Microscopy.

sharon.coe@lehigh.edu

$\checkmark \quad$ Materials Research Society

April 21-25, 2003, San Francisco, CA

info@mrs.org

$\checkmark \quad$ Microscopy and Microanalysis 2003

August 3-7, 2003, San Antonio, TX

www.msa.microscopy.com

$\checkmark \quad$ Society for Neuroscience

November 8-13, 2003, New Orleans, LA web.sfn.org

$\checkmark \quad$ American Society for Cell Biology 2003

December 13-17, 2003, San Francisco, CA

www.ascb.org

$\checkmark \quad$ Materials Research Society

December 1-5, 2003, Boston, MA

info@mrs.org

$\checkmark \quad$ Microscopy and Microanalysis 2004

August $1-5,2004$, Savannah, GA

www.msa.microscopy.com

$\checkmark \quad$ Microscopy and Microanalysis 2005

July 31 -August 4,2005 , Honolulu, HA

www.msa.microscopy.com

Please check the "Calendar of Meetings and Courses" in the MSA journal "Microscopy and Microanalysis" for more details and a much larger listing of meetings and courses.
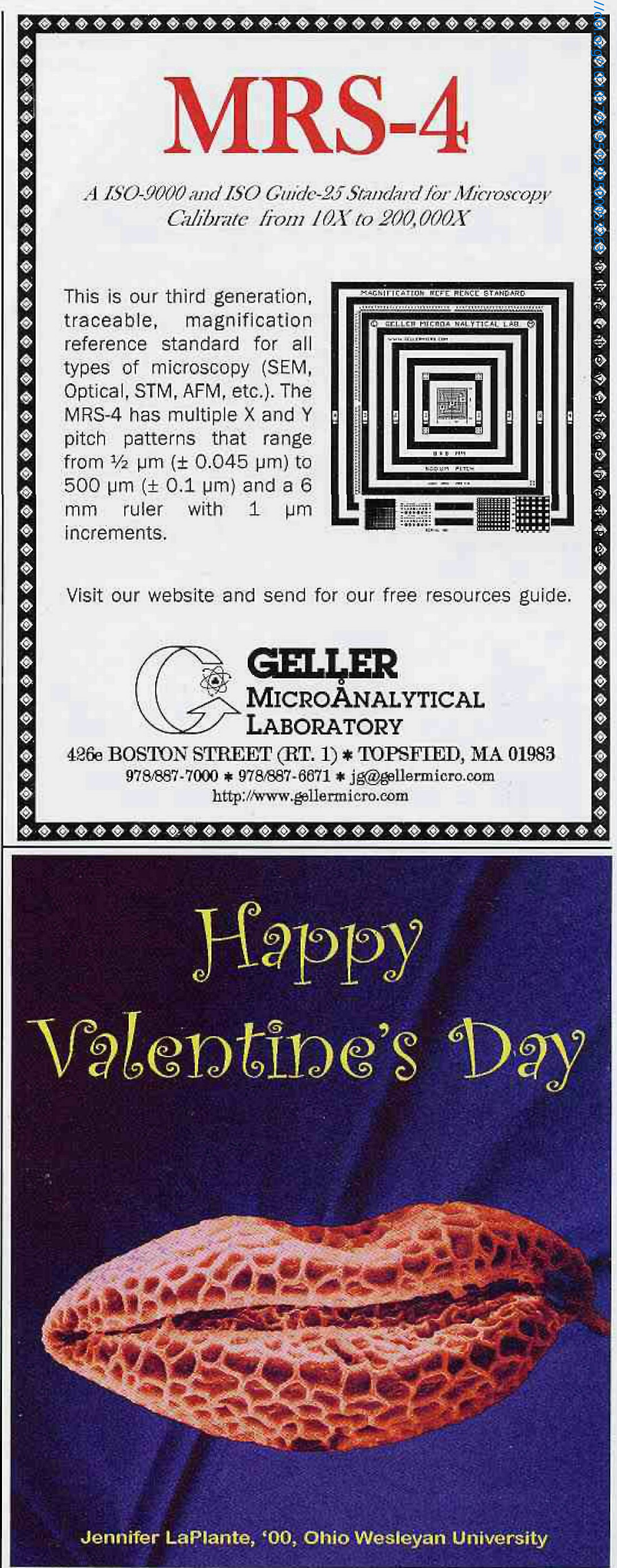

Jennifer is currently in medical school at the University of Florida. Image was taken using a LEO 435VP at the G.W. Burns SEM Laboratory. Submitted by Laura Tuhela-Reuning, OWU. 
Introducing the JSPM-5200 from JEOL, the newest scanning probe microscope to let you explore the structure of surfaces in their native environments.

Analyze samples in fluid at body temperature, investigate optical coatings, and observe magnetic and electrical phenomena.

You control the imaging environment fluid, vacuum, ambient air, or controlled atmosphere and the temperature $(130 \mathrm{~K}-800 \mathrm{~K})$ of your samples.

Powerful image processing and analysis functions plus more than 20 data collection modes - will let you see things you've never seen before. Easy to operate and easy to upgrade, the ISPM-5200 is the most versatile SPM/AFM instrument for your research needs. Contact the imaging experts at JEOL or visit wwwjeol.com/jspm-5200.html to see things as they really are.

\section{Another \\ Extreme Imaging Solution from}

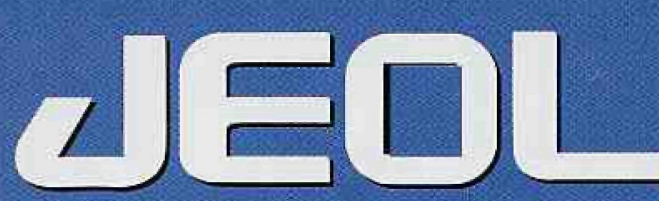

978-535-5900 eod@jeol.com

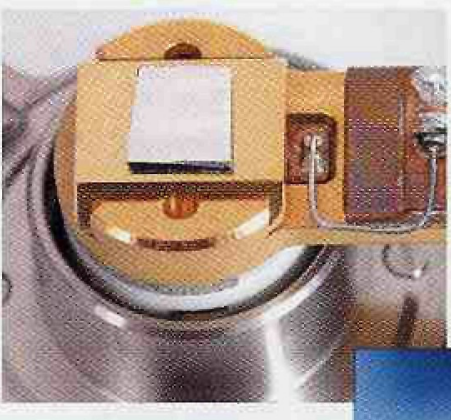

Red Blood Cell Freeze Fractured In Situ

Vacuum level $1 \times 10^{-6}$ tort, 163K. Sample deep etched at $193 \mathrm{~K}$ prior to imaging.

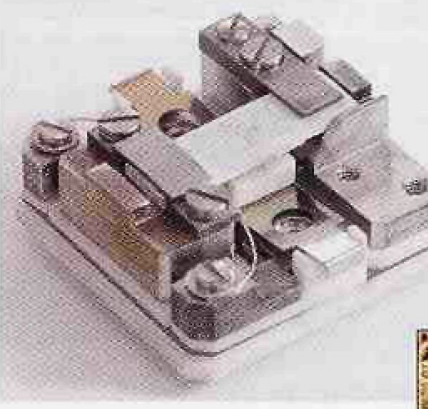

\section{$\mathrm{NaCl}$ at $400^{\circ} \mathrm{C}$}

Time-lapse images

$(360 \mathrm{~s})$ of molecular steps on surface of heated $\mathrm{NaCl}$ crystal. Vacuum level $5 \times 10^{-6}$ torr, $400^{\circ} \mathrm{C}$.
Cold Stage

Liquid nitrogen cooled. Operating temperature rang官 $130 \mathrm{~K}$ to $323 \mathrm{~K}$.

\section{ग.}




\section{Nativel}

\section{of your SPM imaging environment}

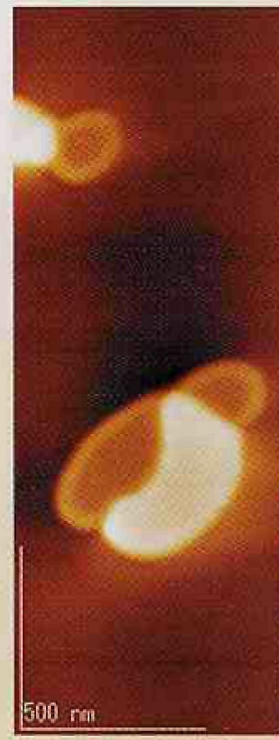

Topography
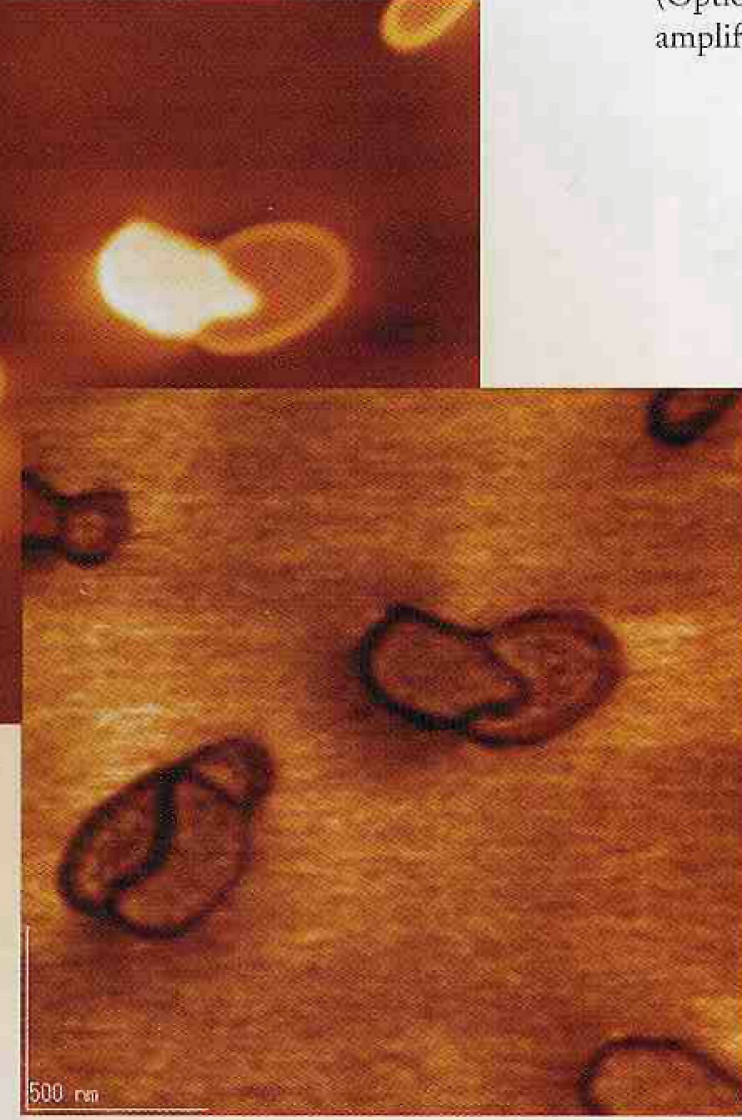

$S K P M$

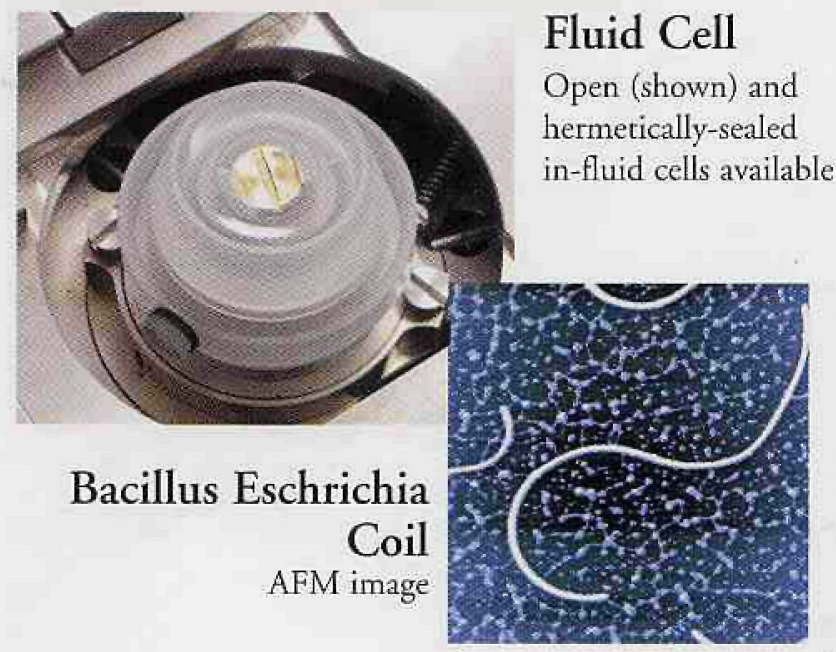

Vacuum Control

$7 \times 10^{-7}$ torr achievable.

(Optional lock-in amplifier shown.)

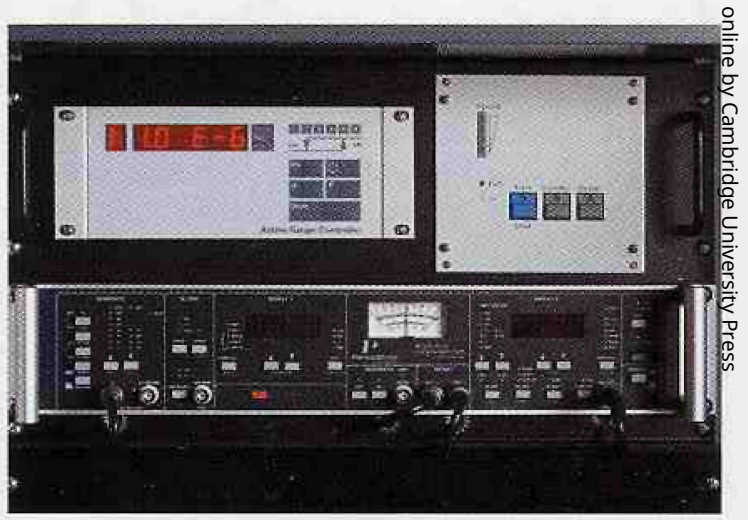

Scanning Kelvin Probe

Microscopy (SKPM)

Images of Sn Alloy

Topography and Kelvin Probe images of tin alloy wire bonding acquired simultaneously. SKPM image shows a reduced capacity to conduct at grain boundaries.

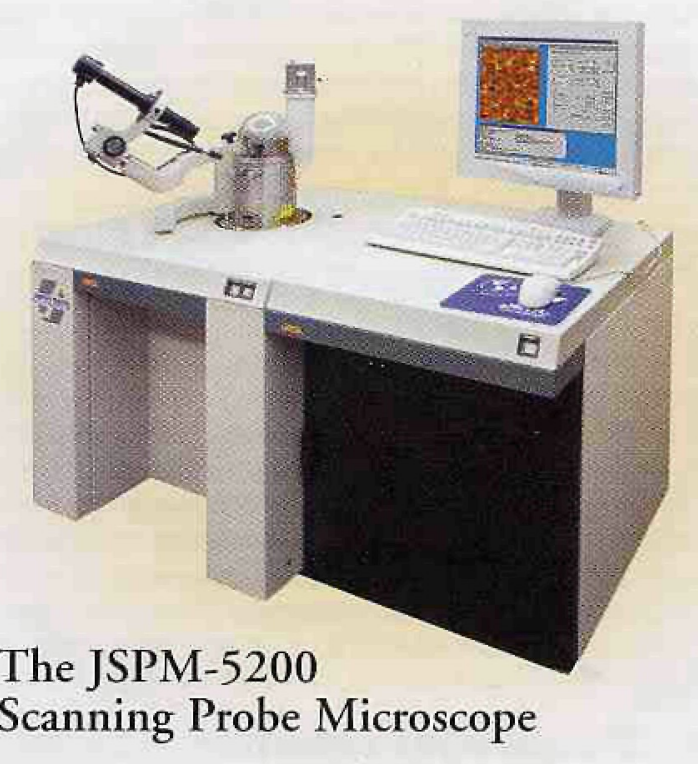




\section{Pt (M)}
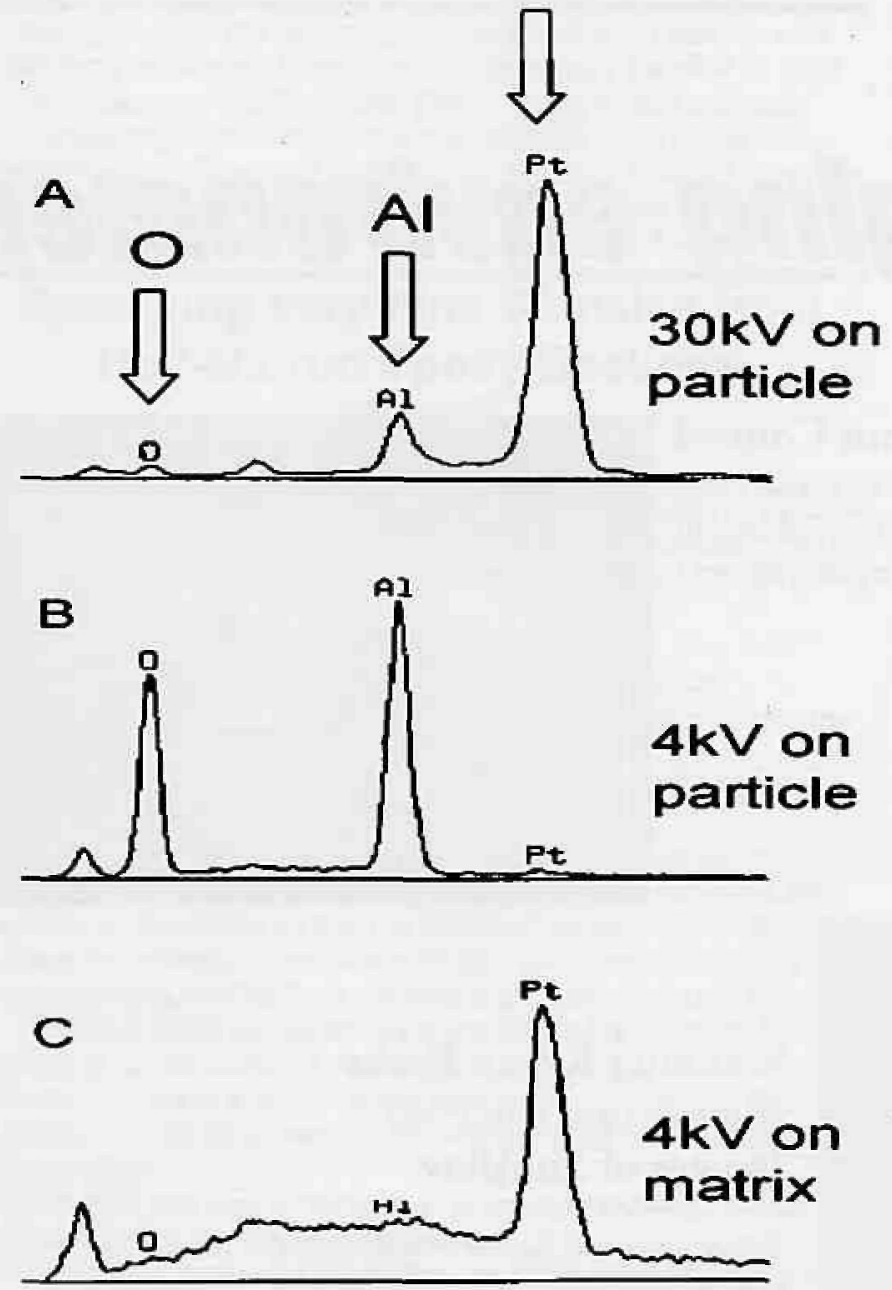

Fig. 5. Analysis of initially unknown particle $\left(\mathrm{Al}_{2} \mathrm{O}_{3}\right)$ in Pt matrix with $k V$

cause peak separations (e.g. $\mathrm{Ta}(\mathrm{M})$ and $\left.\mathrm{Si}(\mathrm{K}), \Delta \mathrm{E}_{\mathrm{x}} \sim 31 \mathrm{eV}\right)$ can be less than the $60-200 \mathrm{eV}$ energy resolution of the $E D X$ detector. Overlap of the $L$ lines of $3 D$ transition elements, such as $C r$, with light element $K$ lines, such as $\mathrm{O}$, is a problem in specific cases, as is the 60-80eV separation of adjacent $3 D \mathrm{~L}$ lines. Classical WDX spectrometers have adequate energy resolution $(-10 \mathrm{eV})$ but their inefficiency in $\mathrm{kcps} / \mathrm{nA}$ sensitivity requires the brutal high current beam conditions of a microprobe which may be specimen destructive for light (and not so light) elements ( 7 ). The advent of $x$-ray optics combined with parallel beam spectrometers (8), and eventually microcalorimeters (9), should help significantly with resolution of this problem. With the correct processing we have shown cold FEG systems can have both the emission stability ( $<1 \% / \mathrm{hr}$ variation) and current $(0.2-1 \mathrm{nA}$ at $5 \mathrm{kV})$ for serial data collection, including spectral imaging maps (fig 3 ).

Fig. 5 is an example of a surface containing an embedded rogue particle. In the high voltage ( $30 \mathrm{kV}$ ) analysis (a) the particle chemistry is mixed up with major contributions from the surrounding matrix, in this case a heat treated Pt EM aperture. By reducing the beam energy to $4 \mathrm{kV}$ the matrix $P t(M)$ line is still adequately excited, if with its usual rather low $\mathrm{P} / \mathrm{B}$ ratio (c) but the analysis on the particle (b) is now contained within the volume of that component. This is established by convergence of the chemistry as the voltage is reduced, since the embedded depth is initially unknown. Even if perfect separation cannot be achieved, the trend allows the component chemistries to be separated. A modern computer controlled instrument can (and should) be operated at a variety of voltages to best effect. The source of the contamination by alumina and other ceramics in this and other $\mathrm{Pt}$ applications may be due to incomplete reprocessing of recycled auto exhaust catalyst materials. A further benefit of the low voltage analysis is the proper representation of the oxygen light element component of the particle chemistry. With a complete forensic characterization it is possible to go look for effective solutions to the problem; or at least methods to ameliorate the negative consequences in key applications. In this case the quick solution was to coat the apertures on both sides with Pt or (much easier) Au-Pd; just like any other recalcitrant high voltage SEM sample.

The challenge now is to turn low voltage analysis into a more general quantitative procedure (10), including (more) reliable parameters for the lower energy $x$-ray lines, and to exploit the potentlal improvements in accuracy; especially for light elements in heavier matrices. The much higher spatial resolution at low voltages helps with one key condition for accurate quantitative analysis: the need to isolate homogeneous analys is volumes in a sample heterogeneous on a fine, sub-micron or nanometer, scale. Almost any SEM can be used for thin film depth analysis. Extending this to three dimensions is assisted by improved instrument configurations. As well as (10) this will be the subject of a future publication.

The characteristics of low voltage analysis include:

- Superior spatial resolution (>10x) and volume sensitivity ( $>1000 \mathrm{x}$ )

- Reduction or elimination of complex $A$ and $F$ matrix corrections at every point

- Proper representation of light elements in spectra - but still no magic for $C$ in $W$ or Si matrices

- Potentially more accurate quantitative analyses, especially of light elements - but a long way to go yet

- Restriction in $x$-ray lines $\left(E_{x}\right)$ and beam energies $\left(E_{0}\right)$ for analyses per element - we use a $130 \%$ minimum ratio for $U=E_{0} / E_{x}$

- Sorne low energy $x$-ray lines have poor fluorescent yields

- $Z$ corrections are more dependent on accelerating voltage $\left(E_{0}\right)$ at low over voltage $(U)$ - but one time determination per system and set up using standards

- Yields and $P / B$ ratios decline with lower $k V$, even for $K$ and $L$ lines - and P/B ratios are already lower for $M$ lines

- Most elements are in principle accessible at $5 \mathrm{kV}$, all of them at $7 \mathrm{kV}$ and a useful number of light and 3D transition elements at $1.5 \mathrm{kV}$ - and this is much more than Castaing had accessible in the first microprobe

- Multiple voltages can be used to deconvolute the depth dimension with trends in convergent chemistry

- New technology spectrometers will transform low voltage analysis by making a wider range of elements accessible through low and very low energy $x$-ray lines, and improving the $P / B$ ratios and analytical sensitivity $10 x$ or more

- Some of the limitations are fundamental but others can be improved with new approaches

- Overall we conclude that low voltage EDX analysis is a powerful new tool. In most, but not all, cases the pros out weigh the cons; especially on the sub-micron scale or for light elements.

The bottom line with low voltage methods is the minimum detectable mass is typically improved by $>100 x$, and the volume sensitivity by $>1000 x$, but for homogeneously distributed material the minimum mass fraction is degraded by up to $10 x$, but usually rather less. The method is best used to analyze the major constituents, especially lo-Z, in small volumes; rather than more widely distributed minor components of heavier elements. A series of very useful new methods have been added to the micro and nano analysis toolbox and they amount to much more than just very high resolution $(-50 \mathrm{~nm}$ ) chemical image $x$-ray maps (useful as they are).

\section{References}

1. R Castaing (1991), in Electron Probe Quantitation, Eds K F J Heinrich and DE Newbury, Plenum, New York.

2. H Bethe (1933), Hdbk. of Physics, Springer, Berlin, Heidelberg and NY

3. E D Boyes (2001), Microscopy and Microanalysis, 6, 307

4. P Echlin (2001), Microscopy and Microanalysis, 7,211

5. E D Boyes (1998), Adv Materials, 10, 1277

6. G Cliff, G W Lorimer (1975), Journal of Microscopy, 103, 203

7. G F Bastin and H J M Heiligers (1991), ibid ref 1.

8. RAgnello, et al. (1997), Proc Microscopy and Micronalysis, 55, 889

9. K D Irwin, D A Wollman (1996), Appl Phys Letts, 69, 1945

10. E D Boyes (2002), Mikrochimica Acta, 138, 225 


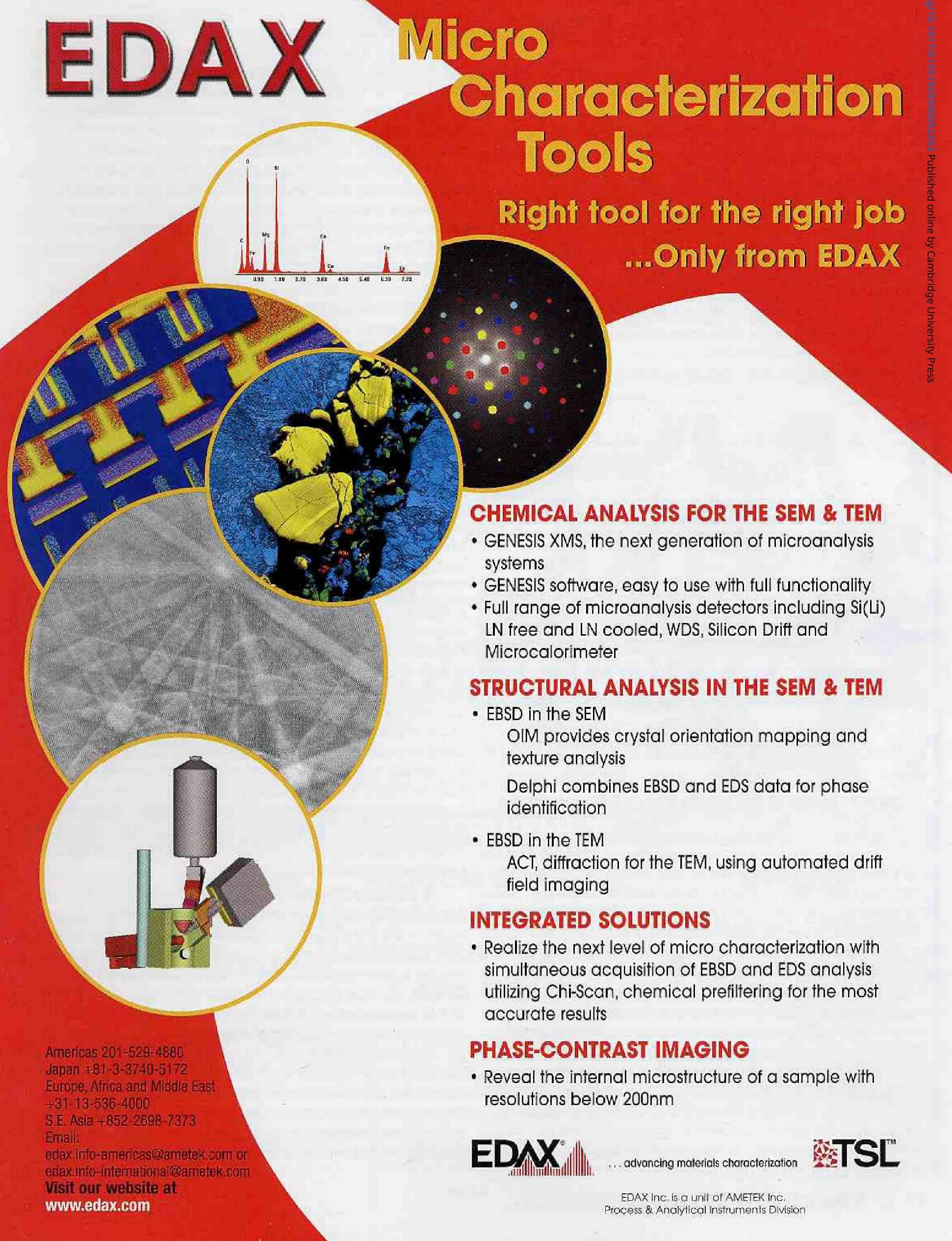

